\title{
Erratum: Distribution of Interface States in MOS Systems Extracted by the Subthreshold Current in MOSFETs under Optical Illumination
}

\author{
[J. Korean Phys. Soc. 43, 873 (2003)] \\ DOI: $10.3938 /$ jkps.43.873
}

\author{
M. S. Kim, H. T. Kim, S. S. Chi, T. E. Kim, H. T. Shin, K. W. Kang, \\ H. S. Park, D. J. Kim, K. S. Min, D. W. Kang and D. M. Kim \\ School of Electrical Engineering, Kookmin University, Seoul 136-702
}

DOI: $10.3938 / \mathrm{jkps} .72 .326$

Sentence (p. 874) in Chapter II starting with "They are ‥ on the device fabrication process [10-15]." should be corrected as "They are $\cdots$ on the device fabrication process [10-15]. We also note that charge states in traps are gate bias-dependent [16].".

Two paragraphs (p. 874) in Chapter II "Figure 2 shows the energy-band diagram … the gate voltage applied across the MOS capacitor." and "Figure 3(c) shows the condition at inversion in which there is now a net negative charge in the acceptor states $[15,16]$." and Figs. 2 and 3 should be removed. 